\title{
ABORDAGEM TEÓRICO-REFLEXIVA DA ESTRATÉGIA DE ENSINO 4E X 2 E SUA INTERFACE COM A TEORIA METACOGNITIVA
}

\author{
César Silva Xavier ${ }^{\text {D1 } 1, ~ M a u r i c i o ~ A b r e u ~ P i n t o ~ P e i x o t o ~ i D 2 ~}$
}

Luciana Lima Albuquerque da Veiga ${ }^{(D)}{ }^{3}$ e Katy C. Cataldo Muniz Domingues 4

\section{Resumo}

Ensaio teórico reflexivo sobre a abordagem metacognitiva de uma estratégia de ensino. 4E X 2 corresponde a uma proposta de instrução por investigação criada por pesquisadores da Clemson University College Education nos EUA na qual 4E significa: Engajar, Explorar, Explicar e Estender e o X2, significa Avaliar e Refletir. O objetivo deste trabalho é apresentar e descrever a 4E X 2 e relacionar seus procedimentos e constructos com a teoria e estratégias de ensino metacognitivo. A descrição detalhada dos procedimentos de aplicação da estratégia fornece subsídios para sua utilização por professores do ensino básico, enquanto sua análise explicita a conexão com a teoria metacognitiva e estratégias de ensino metacognitivas. Observamos aspectos positivos na forma pragmática como os templates e protocolos são apresentados, verificamos interfaces entre a estratégia e os componentes do conhecimento e do controle executivo autorregulador, assim como, os procedimentos de aplicação apresentam-se compatíveis com as definições das funções das estratégias de ensino metacognitivo. Este estudo reforça a importância da aproximação da produção do contexto acadêmico com o ambiente escolar, onde o principal objetivo sempre será a reprodução possível destas teorias na prática do dia a dia do professor.

Palavras-chave: Estratégias; Ensino; Metacognição; Instrução.

\section{THEORETICAL-REFLECTIVE APPROACH OF THE 4E X 2 TEACHING STRATEGY AND ITS INTERFACE WITH THE METACOGNITIVE THEORY}

\footnotetext{
Abstract

Reflective theoretical essay on the metacognitive approach of a teaching strategy. 4E X 2 corresponds to an inquiry instruction proposal created by researchers at Clemson University College Education in the USA in which 4E stands for: Engage, Explore, Explain and Extend and X2 stands for Evaluate and Reflect. The aim of this paper is to present and describe 4E X 2 and relate its procedures and constructs to metacognitive theory and teaching strategies.

${ }^{1}$ Doutorando em Educação em Ciências e Saúde. Universidade Federal do Rio de Janeiro e Professor EBTT do Instituto Federal do Espírito Santo (IFES)

${ }^{2}$ Doutor em Medicina pela Universidade Federal do Rio de Janeiro (UFRJ). Professor da UFRJ.

${ }^{3}$ Doutoranda em Educação em Ciências e Saúde pela Universidade Federal do Rio de Janeiro (UFRJ).

${ }^{4}$ Doutoranda em Educação em Ciências e Saúde pela Universidade Federal do Rio de Janeiro (UFRJ).
} 
The detailed description of the strategy's application procedures provides subsidies for its use by basic education teachers, while its analysis explains the connection with metacognitive theory and metacognitive teaching strategies. We observed positive aspects in the pragmatic way in which templates and protocols are presented, we verified interfaces between the strategy and knowledge components of knowledge and self-regulating executive control, as well as the application procedures are compatible with the definitions of the functions of the strategies of metacognitive teaching. This study reinforces the importance of bringing production from the academic context closer to the school environment, where the main objective will always be the possible reproduction of these theories in the daily practice of the teacher.

Keywords: Strategies; Teaching; Metacognition; Instruction.

\section{Introdução}

Atualmente há na pesquisa em ensino de Ciências uma tendência pela formação de indivíduos autônomos, com habilidades para monitorar e regular seus próprios processos de aprendizagem, especialmente através da solução de problemas (ROSA; VILAGRÁ, 2020).

Neste sentido reconhecemos a importância de pensarmos em estratégias capazes de mobilizar professores para um ensino orientado com 0 objetivo de desenvolver no aprendiz o autoconhecimento e a habilidade para aprender a aprender e aprender de forma eficiente (CLEOPHAS; FRANCISCO, 2018).

Sob esta perspectiva acreditamos que metacognição seja capaz de fornecer ferramentas para a melhora do trabalho do professor na tarefa de ensinar de modo a estimular no aluno a reflexão e o raciocínio sobre os modos pelos quais realiza uma atividade ou durante a resolução de uma situação problema (CLEOPHAS; FRANCISCO, 2018; LEITE; DARSIE, 2011).

Assim apesar de alguns alunos recorrerem a estratégias metacognitivas de aprendizagem mesmo que não percebam (ROSA; ROSA, 2016), a aquisição destas habilidades não ocorre de forma igual em todos aos aprendizes. Segundo Locatelli (2014), isto exige esforço, tomada de consciência e reavaliação constante daquilo que está fazendo. Neste sentido, Chi et al (1989) consideram necessário que o pensamento metacognitivo seja inserido de forma explícita nos processos educacionais. Pois, segundo Rosa e Alves Filho (2014), para que os alunos tenham a capacidade de recorrer ao seu pensamento metacognitivo, é necessário inicialmente ativá-lo.

A literatura científica tem demonstrado o uso de Estratégias de Ensino Metacognitivo (MARAGLIA, 2018) com o intuito de introduzir e reforçar nos aprendizes o pensamento metacognitivo durante o aprendizado de conteúdos científicos. (MARAGLIA, 2018; ROSA; VILLAGRÁ, 2018; XAVIER; PEIXOTO, 2019; XAVIER; PEIXOTO; VEIGA, 2020). 
Neste contexto, apresentamos aqui um recorte de pesquisa de doutorado, que tem o objetivo de avaliar e comparar os aspectos da teoria metacognitiva e a possibilidade de aplicação de diferentes Estratégias de Ensino Metacognitivo (EEM) no ambiente escolar.

Especificamente para este estudo, nos dedicamos à análise da instrução denominada 4E X 2 (leia-se $4 \mathrm{E}$ por 2) visando tomar conhecimento dos passos necessários para a sua execução e analisar as relações entre os constructos da estratégia com os aspectos teóricos da metacognição e das estratégias de ensino metacognitivas.

Para tanto este estudo tem como questões norteadoras: Como se dá o percurso de aplicação? Quais são os constructos envolvidos na 4E X 2? Como a metacognição está inserida? Desta forma nossos esforços se concentraram em descrever os procedimentos de aplicação da estratégia e analisar a abordagem metacognitiva sob a Luz da teoria metacognitiva.

Assim o texto foi organizado da seguinte maneira: I. Fundamentação teórica da metacognição e estratégias de ensino metacognitivas; II. Percurso metodológico; III. Resultados, onde apresentamos os constructos da 4E X 2 e a descrição das etapas de aplicação com o objetivo de fornecer uma orientação ao leitor interessado em produzir uma estratégia baseada na 4E X 2 . IV. Discussão, onde apontamos as relações entre os constructos da $4 \mathrm{E} X 2$, as referencias teóricas sobre metacognição e estratégias de ensino metacognitivo; V. Considerações finais.

Observamos que os procedimentos de aplicação podem ser reproduzidos a partir da sua descrição. E verificamos a relação entre os constructos da 4E X 2 e tópicos da teoria metacognitiva e das estratégias de ensino metacognitivo no que tange a possibilidade de estímulo no aprendiz, para o exercício de conhecer sobre o que ele conhece e para planejar, monitorar e avaliar a tarefa que está desenvolvendo.

\section{Metacognição}

Originalmente a metacognição foi identificada como conhecimento e regulação das atividades cognitivas de uma pessoa nos processos de aprendizagem (BROWN, 1978; FLAVELL, 1979).

Segundo Flavell; Miller e Miller (1999), a metacognição é um termo de definição ampla e livre "como qualquer outro conhecimento ou atividade cognitiva que toma como seu objeto, ou regula, qualquer aspecto de qualquer iniciativa cognitiva" (p. 125). Apesar de aparentemente simples, o termo metacognição tem se apresentado na literatura de forma complexa, difusa e obscura, sendo detalhada especificamente de acordo com a área de investigação (ROSA 2011).

Assim adotamos para a metacognição as definições propostas pelo precursor, o psicólogo John Hurley Flavell na década de 1970 juntamente com as descrições e detalhamentos oriundos dos estudos de do psicólogo Henry 
Wellman e da psicóloga Ann Brown. A organização conceitual e funcional da metacognição está ancorada nas propostas de Rosa (2011) e Rosa e Villagrá (2020). Neste contexto a metacognição pode ser definida como

Conhecimento que o sujeito tem sobre seu conhecimento e a capacidade de regulação dada aos processos executivos, somada ao controle e à orquestração desses mecanismos. (ROSA; VILLAGRÁ 2020, p. 62).

Apoiada nas contribuições de Flavell, Wellman e Brown, Rosa (2011) propõe uma aproximação entre a Educação em Ciências e as componentes metacognitivas (conhecimento do conhecimento e o controle executivo regulador) introduzidos por Flavell (1976). A primeira componente, conhecimento metacognitivo ou conhecimento do conhecimento corresponde à crença da qual um indivíduo possui sobre si mesmo, fica armazenado na memória a partir de experiências, atua sobre os processos cognitivos sendo passível de verbalização e acesso. Esta componente diz respeito ao que o indivíduo conhece sobre o próprio conhecimento e, segundo Rosa e Villagrá (2020, p. 713), "o uso que faz dele para aprender como realizar a busca no mundo externo".

Detalhada por Flavell e Wellman (1977), a primeira componente abrange três variáveis ou elementos metacognitivos: pessoa, tarefa e estratégia. A variável Pessoa representa as qualidades pessoais, abrange o que você pode acreditar a respeito do que conhece sobre a própria natureza e a respeito do que conhece sobre a natureza de outras pessoas. Pode ser intrapessoal quando diz respeito ao próprio indivíduo, ou interpessoal quando diz respeito a outrem. A variável Tarefa está relacionada ao conhecimento sobre a natureza da informação, por exemplo, se o conteúdo é familiar ou desconhecido, bem ou mal organizado, confiável ou não. E a variável Estratégia indica quais são os processos, procedimentos e ações que permitem que o indivíduo atinja seus objetivos com maior eficácia. Para Rosa e Villagrá (2020, p. 62).

A variável "Pessoa" relaciona-se às convicções, crenças e mitos que o indivíduo possui acerca de sua própria cognição, inclusive estabelecendo comparações de si mesmo com os outros. A varável "Tarefa" está associada às características da atividade em andamento, tanto em termos do que ela é como do que envolve, como por exemplo a forma como o sujeito se identifica frente a determinada atividade como a leitura ou o manuseio de equipamentos de medição. A variável "Estratégia", por sua vez, vincula-se ao como e quando fazer, estando relacionada ao momento que em que o sujeito se questiona sobre o que precisa ser feito e quais os caminhos a serem seguidos para atingir o objetivo.

A segunda componente, controle executivo autorregulador, está relacionada à capacidade de planejar, verificar a necessidade de adequação e 
fazer os ajustes necessários para atingir o objetivo (BROWN, 1978; FLAVELL, 1979). Ela compreende os elementos planificação, monitoração e avaliação.

A planificação envolve a capacidade da criança planejar, prever etapas, escolher estratégias de acordo com o objetivo, além da capacidade de planejar com antecedência, a planificação envolve o conhecimento sobre a eficiência de tal planejamento. A monitoração está relacionada ao controle da ação, pode incluir revisão, verificação e percepção de erros de modo que se possa e corrigi-las quando necessário a fim de alcançar o objetivo pretendido. E a avaliação consiste na verificação dos resultados alcançados em função dos objetivos inicialmente propostos (BROWN, 1978; 1987). Segundo Rosa e Villagrá (2020, p. 63).

A "Planificação" é a responsável pela previsão de etapas e escolha de estratégias em relação ao objetivo pretendido, o que supõe fixar metas sobre como proceder para realizar a ação. O planejamento inicial representa as decisões do sujeito em relação ao que vai ser executado, consistindo em ações independentes e decorrentes de decisões influenciadas pelo conhecimento do sujeito. O "Monitoramento" consiste em controlar a ação e verificar se está adequada para atingir o objetivo proposto, avaliando o desvio em relação a este, percebendo erros e corrigindo-os, se necessário. A "Avaliação", por sua vez, está identificada com os resultados atingidos em face do fim visado, podendo, eventualmente, ser definida pelos critérios específicos de avaliação. No âmbito escolar, esse é o momento em que os estudantes retomam e avaliam a aprendizagem com o intuito de identificar como a realizaram.

A funcionalidade da proposta que envolve os componentes e elementos metacognitivos é complementada pela ferramenta didática dos questionamentos metacognitivos (ROSA, 2011; ROSA; VILLAGRÁ, 2020), representados na tabela 1 .

Esta proposta apresenta interface com estratégias que utilizam prompts ou comandos metacognitivos que funcionam na orientação dos alunos para conduzir de forma mais eficaz a própria aprendizagem e a solução de problemas (PETERS; KITSANTAS, 2010) como é o caso da 4E X 2. 
Tabela 1: Questionamentos metacognitivos

\begin{tabular}{|c|c|c|}
\hline & $\begin{array}{c}\text { Elementos } \\
\text { metacognitivos }\end{array}$ & Perguntas metacognitivas \\
\hline \multirow{3}{*}{ 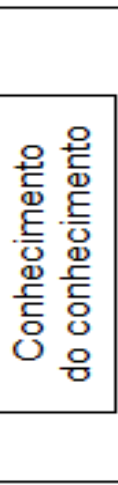 } & Pessoa & $\begin{array}{l}\text { Identifica este assunto com outro já estudado? Qual o sentimento em relação } \\
\text { a este conhecimento? Compreendeu a atividade? Está interessado em } \\
\text { realizar a atividade proposta? Apresenta conhecimento sobre o assunto? } \\
\text { Apresenta limitações neste tema? }\end{array}$ \\
\hline & Tarefa & $\begin{array}{l}\text { Entendeu a tarefa? Que tipo de tarefa é essa? Identifica-a com outra já } \\
\text { realizada? Julga ter facilidade ou dificuldade em realizar tarefas como a } \\
\text { proposta? Está de acordo com seus conhecimentos? Identifica o que é } \\
\text { preciso para resolvê-la? }\end{array}$ \\
\hline & Estratégia & $\begin{array}{l}\text { Conhece estratégias para resolver este tipo de problema? Tem facilidade } \\
\text { com este tipo de estratégia? Há outras possibilidades de realização da } \\
\text { tarefa? Dispõe do que precisa para executar a tarefa? } \\
\text { Controle executivo e autorregulador }\end{array}$ \\
\hline \multirow{3}{*}{ 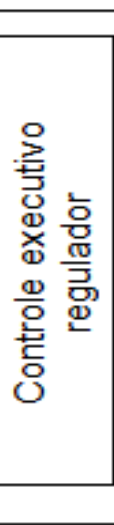 } & Planificação & $\begin{array}{l}\text { O que entendeu sobre a atividade proposta? Identifica por onde deve } \\
\text { iniciar? Como organizar as informações apresentadas na atividade? } \\
\text { Consegue visualizar o procedimento em relação ao fim almejado? }\end{array}$ \\
\hline & Monitoração & $\begin{array}{l}\text { Compreende bem o que está fazendo? Qual o objetivo desta atividade? A } \\
\text { estratégia que utiliza é adequada? Tem domínio do que está executando? Há } \\
\text { necessidade de retomar algo? O planejado está funcionando? Por que está } \\
\text { realizando a atividade proposta? Continuando desta forma, vai atingir os } \\
\text { objetivos dessa atividade? }\end{array}$ \\
\hline & Avaliação & $\begin{array}{l}\text { Consegue descrever o que realizou e como realizou? Qual era o objetivo } \\
\text { proposto inicialmente? Houve necessidade de rever algo durante a } \\
\text { realização da atividade? Qual o resultado da atividade? Tem consciência do } \\
\text { conhecimento adquirido com a realização da atividade? Os resultados } \\
\text { encontrados foram os esperados? }\end{array}$ \\
\hline
\end{tabular}

Fonte: Adaptado de Rosa e Villagrá (2020).

\section{Metacognição e Estratégias de ensino metacognitivo}

Conforme descrito nas linhas anteriores, uma das alternativas para a formação de alunos autônomos e críticos sobre suas condições de aprendizagem é o uso da metacognição. Segundo Locatelli (2014), a metacognição envolve uma variedade de processos abrangendo o monitoramento e o repensar sobre os próprios conhecimentos. Estes processos levam de forma gradativa a um aumento na autonomia para estudar e aprender.

No entanto, embora os processos metacognitivos possam ser usados para o controle das estratégias de estudo, este processo pode não ocorrer de forma espontânea (DUNLOSKY; HERZOG, 1998). Para que o pensamento metacognitivo do aluno seja ativado, faz-se necessário o desenvolvimento e aprimoramento de alternativas didáticas que subsidiem as ações do professor (ROSA; ALVES, 2014). Dentre as alternativas didáticas, temos pesquisado as Estratégias de Ensino Metacognitivo (EEM) que segundo Maraglia (2018) correspondem a

Ações pedagógicas planejadas e empregadas pelo professor que, além de trabalhar conteúdos, potencializem a autoconsciência de alunos em contextos de aprendizagem, propondo um discurso de segundo nível sobre a cognição, promovendo assim, a aprendizagem autorregulada". (MARAGLIA, 2018, p. 35). 
Estudos desenvolvidos por diversos autores (CLEARY; PLATTEN, 2013; OSES; CARRASCO, 2013; ROSA; ALVES FILHO, 2014; ROSA; VILLAGRÁ, 2018; XAVIER; PEIXOTO; VEIGA, 2020) apontam benefícios relacionados ao uso de EEM. Neste sentido, temos observado estratégias didáticas instrucionais com bases na metacognição, propostas e/ou aplicadas sob diferentes perspectivas e metodologias, todas elas implementando o ensino de algum conteúdo científico vinculado ao pensamento metacognitivo de modo a levar o aluno à reflexão e identificação de mecanismos que favoreçam sua própria aprendizagem.

\section{Percurso metodológico}

Pesquisa com abordagem qualitativa e paradigma de pesquisa crítica (Esteban, 2010). Este estudo tem o objetivo de produzir um ensaio teóricoreflexivo sobre a relação entre a $4 \mathrm{E} \times 2$, os aspectos da teoria metacognitiva e as estratégias de ensino metacognitivo.

A fonte primária deste estudo foi o artigo teórico, $4 E \times 2$ Instructional Model: Uniting Three Learning Constructs to Improve Praxis in Science and Mathematics Classrooms de Jeff C. Marshall; Bob Horton e Julie Smart (2008).

O processo da pesquisa foi realizado em diferentes fases e níveis de leitura. Realizamos a princípio a leitura inspecional, ou pré-leitura (ADLER; DOREN, 2010). Esta entendida como um nível de leitura superficial procurando extrair as informações iniciais, visando rapidez, reconhecimento da estrutura do texto e identificação de elementos sugestivos de leitura mais profunda. (PRATES; BETARELLO; FINELLI, 2016)

A leitura exaustiva do material concentrou-se na teoria metacognitiva, nos constructos da instrução por investigação, da reflexão metacognitiva, da avaliação formativa e nos procedimentos de aplicação da estratégia.

A partir da identificação dos elementos acima citados, passamos a executar a Leitura Analítica. Esta definida como uma leitura mais completa e aprofundada utilizando o tempo necessário para a compreensão do texto. Posteriormente realizamos a Leitura Sintópica ou comparativa, neste caso, utilizando e comparando outras referências que tratam do mesmo assunto. (ADLER; DOREN, 2010)

Estes dois últimos níveis de leitura se concentraram especialmente, conforme dito anteriormente, na teoria metacognitiva, nos constructos da instrução por investigação, na reflexão metacognitiva, na avaliação formativa e nos procedimentos de aplicação da 4E X 2.

Posteriormente apresentamos os resultados com a seguinte organização: I. O tópico Compreendendo a $4 E \times 2$, apresenta e descreve os constructos da 4E X 2; II. O tópico Visão geral e descrição do modelo, apresenta os procedimentos de aplicação com o objetivo de possibilitar sua reprodução no ambiente escolar; III. No tópico $A$ metacognição na $4 E X 2$, buscamos relacionar cada elemento da estratégia $4 \mathrm{E} X 2$ com a teoria metacognitiva de Flavell, Wellman e Brown, com as questões metacognitivas 
de Rosa (2011); Rosa e Villagrá (2020) e com as definições e características das estratégias de ensino metacognitivas.

\section{Compreendendo a 4E X 2}

Apresentamos aqui a uma síntese da EEM 4E X 2. Pretendemos destacar os aspectos teóricos abordados nesta EEM. Neste caso dedicamos nossa atenção para a relação entre teoria metacognitiva e os constructos necessários e utilizados em seu desenvolvimento. Além disso, descrevemos os passos necessários para a sua realização, de modo a auxiliar os leitores interessados em sua reprodução.

O $4 E \times 2$ Instructional Model é apresentado como um modelo instrucional teórico desenvolvido na Clemson College Education nos EUA. Consiste em uma estratégia que utiliza três constructos de forma integrada para vincular a compreensão conceitual e a aprendizagem por investigação. Os constructos de aprendizagem integrados nesta estratégia são I. Avaliação formativa, II. Modelo instrucional de investigação (ensino baseado na investigação) e III. Reflexão metacognitiva. Estes constructos serão apresentados e discutidos mais adiante.

Marshall, et al (2008) consideram que apesar da existência de outros modelos instrucionais capazes de imergir estudantes em aprendizagem baseada em investigação, nenhum deles aborda de forma explícita a importância da avaliação formativa e da reflexão metacognitiva. Estes são necessários em cada estágio da aprendizagem.

Para situar o leitor, dedicamos os próximos três tópicos para descrever individualmente os constructos que orientam na $4 \mathrm{E} \times 2$ de modo permitir uma aproximação com os fundamentos da estratégia.

\subsection{Reflexão metacognitiva}

Apesar de a literatura utilizar frequentemente os termos metacognição e reflexão de forma intercambiável, Wilson e Clarke (2004) não consideram que estes termos correspondam a sinônimos. Para os autores a prática reflexiva se refere a qualquer ocasião de pensamento intencional, não necessariamente sobre os próprios pensamentos.

O pensamento reflexivo é uma estratégia cognitiva que possibilita a compreensão mais profunda de fenômenos e processos, por meio da percepção das relações, da identificação dos elementos envolvidos, da análise e interpretação dos sentidos e significados, potencializando o processo de aprendizagem. (GASQUE, 2011, p. 40).

Por outro lado, a metacognição abrange a compreensão e o controle que um indivíduo possui a respeito de seus próprios processos cognitivos e inclui a análise e a consciência dos processos centrais de pensamento de modo 
a contribuir com uma aprendizagem efetiva. (STERNBERG, 1998; WHITE; FREDERIKSEN, 2005; MARSHALL ET AL, 2008).

Desta forma, podemos admitir que a metacognição pode assumir o conceito de "algo como pensar sobre o seu pensamento ou seu conhecimento sobre as coisas" (LOCATELLI, 2014, P. 23), ou como anunciam Peixoto et al, (2007, p. 69 e 70), "uma cognição sobre a cognição ou atributo cognitivo ou conhecimento sobre o fenômeno cognitivo. Sendo, portanto, um discurso de segundo nível sobre o conhecimento."

Neste sentido, podemos afirmar que a reflexão está relacionada com quase todas as circunstâncias de pensamento. Diferentemente a metacognição inclui uma análise mais profunda e consciência dos próprios pensamentos. (MARSHALL ET AL, 2008)

A 4E X 2 propõe que a reflexão metacognitiva ocorre a partir da unificação entre a prática reflexiva que se apresenta focada nos conceitos investigados e as perspectivas da autoconsciência asseguradas pela metacognição. (STERNBERG, 1998)

Esta perspectiva de intercâmbio entre reflexão e metacognição também é descrita por Tarricone (2011) e corresponde a um evento de longa data. Esta autora, apoiada em filósofos como Aristóteles, Agostinho e Descartes e teóricos como Baldwin, Dewey, Spearman, considera que a autoconsciência é desenvolvida e instigada pela reflexão, estimulada por contextos de resolução de problemas. Assim, os aspectos da autorregulação da reflexão são essenciais para a metacognição.

Professores em atividade que refletem sobre suas práticas apresentam melhores resultados, uma vez que seus alunos obtém melhor desempenho acadêmico (VANDEVOORT ET AL, 2004). No entanto é fundamental destacar que se a prática reflexiva se detiver apenas entre os profissionais, certamente haverá perda na oportunidade de aprendizagem (MARSHALL ET AL, 2008). Isto significa que é de muito importante que os professores criem oportunidades para que os aprendizes possam conhecer e experimentar a reflexão sobre suas práticas e sobre seus procedimentos de aprendizagem. (LOCATELLI, 2014)

Este entendimento corrobora com a sugestão de que alunos que se envolvem em reflexões bem orientadas ao longo de um processo de aprendizagem, apresentam ganhos significativos (ASCHBACHER; ALONZO, 2004; SHEPARDSON; BRITSCH, 2001). Desta forma, ajudar o estudante a refletir sobre o que foi aprendido e sobre o que ainda necessita aprender, pode melhorar sua capacidade cognitiva para direcionar seu aprendizado no futuro.

\subsection{Avaliação Formativa}

Os processos avaliativos se configuram como atividades complexas, envolventes e instigantes. Ao longo do tempo, constata-se a coexistência de diferentes tipos de avaliação no ambiente acadêmico. Ora a atividade avaliativa se configura como importante aliado no processo ensinoaprendizagem, ora como instrumento reprodutor, disciplinador e classificatório (c) (1) $(2)$ 
e ora como uma atividade confusa, perdida entre esses dois polos. (VIEIRA; LIMA, 2019).

Dentre vários tipos de avaliação descritos na literatura, destacamos aqui dois: a avaliação somativa e a avaliação formativa. A avaliação somativa, largamente utilizada nos mais variados níveis educacionais, caracteriza-se pela verificação das "capacidades, competências e habilidades" do aluno, sua turma, escola e/ou seu sistema educacional ao final de uma etapa formal. Por outro lado, a avaliação formativa ocorre ao longo do processo e tem como objetivo "identificar inadequações, sugerir melhorias para replanejamento e redesenvolvimento de ações", de modo que se obtenha um resultado final, próximo do esperado (ANDRIOLA; ARAÚJO, 2018, p. 7).

Conscientes da complexidade dos temas relacionados à avaliação no ambiente escolar e acadêmico, assim como na pesquisa primária, não pretendemos aprofundar os diversos tipos de processos avaliativos. Desta forma nos detivemos, neste tópico, a apresentar especificamente a avaliação formativa utilizando os conceitos dos teóricos abordados e necessários para 0 desenvolvimento da EEM 4E X 2.

Apesar da presença de diferentes tipos de avaliação descritos na literatura, a avaliação formativa foi o tipo adotado como constructo na EEM 4E X 2. Com base nas ideias de Black e Willian (1998) os autores da pesquisa primária afirmam que a avaliação formativa engloba toda e qualquer atividade realizada por professores e alunos, capaz de fornecer informações que possam ser utilizadas como feedback para modificar as atividades de ensino e aprendizagem às quais estão empenhados. No mesmo sentido Andriola e Araújo (2018, p.7) afirmam que o que uma:

[...] autêntica avaliação formativa tenta responder é: Por que não avaliar os alunos para informá-los a tempo sobre os seus erros, sobre como se deve estudar e sobre o que se deve estudar, sem esperar a conclusão de uma fase ou etapa da educação formal?

Estudos de Black e Wiliam (1998) apontam que a utilização de avaliação formativa tomada como parte do processo apresenta efeitos positivos e significativos aos alunos durante seus processos de aprendizagem. Desta forma este tipo de avaliação pode atuar no encorajamento ao aprendizado de modo a estimular a busca pelo conhecimento, não como produto acabado, mas de forma integrada ao processo de aprendizagem.

Assim, diante de tais perspectivas, Vieira e Lima (2019) sugerem que avaliação formativa se apresenta com o caráter de aliado ao processo de ensino-aprendizagem, especialmente se considerarmos que ela é aplicada ao longo do processo, podendo influenciar nas ações adotadas pelos professores e alunos durante o desenvolvimento das atividades. 


\subsection{Modelos instrucionais de investigação}

Outro constructo da 4E X 2 é o do Modelo Instrucional de Investigação. Modelos instrucionais são orientações ou estratégias às quais se baseiam as abordagens utilizadas pelos professores na tarefa de ensinar. Quando se fala em modelo instrucional de investigação, significa que as orientações e estratégias utilizadas pelos professores estão direcionadas a processos investigativos que são próprios da atividade científica.

Com base nas definições do Conselho Nacional de Pesquisa (National Research Council - NRC) dos EUA, Marshall et al (2008) define investigação como um conjunto de processos inter-relacionados em que pesquisadores e estudantes levantam questões sobre 0 mundo natural e buscam 0 entendimento sobre fenômenos. Ao realizarem investigações, os estudantes podem adquirir conhecimento e desenvolver compreensão de conceitos, princípios modelos e teorias referentes ao mundo que os rodeiam.

No entanto, esta definição para investigação pode ser considerada incompleta quando nos referimos ao constructo "Modelo Instrucional de Investigação". Assim uma definição mais apropriada e adequada para um modelo instrucional de investigação seria a de "currículos e práticas instrucionais que promovem e facilitam o engajamento dos estudantes na ideia de investigação científica" (MARSHALL ET AL, 2008, p. 504). Logo, em um modelo instrucional de investigação, os alunos são orientados no aprendizado do conteúdo através de procedimentos utilizados em investigação.

A inserção dos componentes de avaliação formativa e reflexão metacognitiva, encontrados no 4E X 2 buscam a ampliação do paradigma instrucional, auxiliando o desenvolvimento de habilidades para que professores possam facilitar o aprendizado baseado em investigação de forma mais profunda e significativa.

\subsection{Visão geral e descrição do modelo}

Este tópico tem o objetivo de apresentar os procedimentos de aplicação da EEM 4E X 2 e orientar os leitores interessados em reproduzir a estratégia junto às suas turmas.

$\mathrm{Na}$ inscrição 4E X 2 o 4E representa a instrução por investigação e corresponde às iniciais dos termos Engage, Explore, Explain e Extent respectivamente (Engajar, Explorar, Explicar e Estender) e o 2 refere-se aos termos Reflect e Assess (Reflexão e Avaliação). Consiste em um modelo de instrução que promete utilizar experiências de aprendizagem por investigação associadas à avaliação formativa e reflexão metacognitiva para a construção de conceitos científicos.

Apesar do artigo 4E $\times 2$ Instructional Model: Uniting Three Learning Constructs to Improve Praxis in Science and Mathematics Classrooms se tratar de uma discussão de natureza teórica, a Clemson Collegge of Education fornece propostas de aplicação do 4E X 2 no ensino fundamental, médio e 
superior nas disciplinas de matemática e das ciências da natureza. As propostas são apresentadas na forma de sequências didáticas apropriadas para o desenvolvimento em sala de aula e/ou laboratório de ciências e/ou laboratório de informática.

O modelo aqui descrito oferece aos professores um sistema dinâmico que orienta o desenvolvimento e a implementação de oportunidades de aprendizado com o estabelecimento de reflexão metacognitiva e avaliação formativa ao longo do processo. De forma envolvente e interativa a sequência de passos propostos pelo modelo instrucional 4E X 2 exige dos alunos que façam uma pausa, pensem, interajam, reflitam e registrem seus padrões de solução. Assim, professor e aluno são ativos na situação de ensino/aprendizagem. O aluno reflete e avalia o processo cujo tema abordado foi minunciosamente planejado e implementado pelo professor.

A Figura 1 fornece uma visão geral de como os três principais construtos (reflexão metacognitiva, modelo instrucional de investigação e avaliação formativa) se inter-relacionam. Repare que as laterais do esquema apresentam à esquerda o termo Avaliar (Assess) que representa a Avaliação Formativa e à direita o termo Refletir (Reflect) que representa a reflexão metacognitiva. Ressaltamos que os termos Avaliar e Refletir não são fases fixas, mas fluidas de modo que ocorra ao longo de todo o processo, interpassando pelas diferentes fases Engajar, Explorar, Explicar e Estender do modelo.

Figura 1: Interação dos três constructos no 4E X 2.

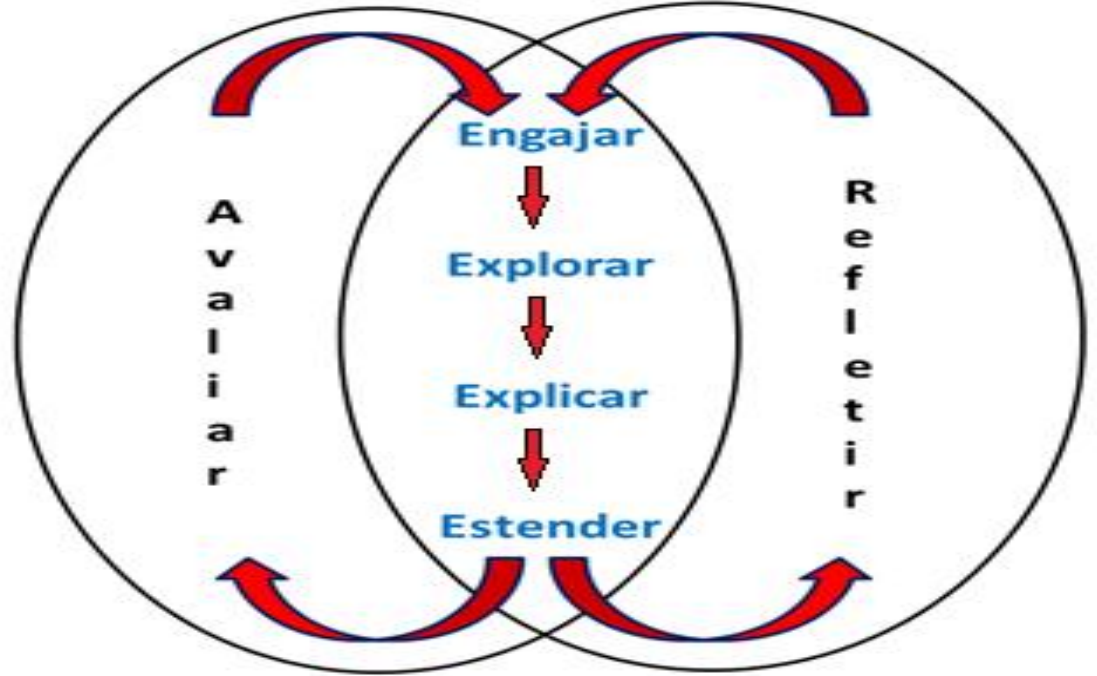

Fonte: Adaptado de Marschall (2008)

O plano de execução do 4E X 2 segue momentos ou fases específicas de acordo com o tema escolhido pelo professor. O eixo central do esquema representa o constructo do modelo instrucional de investigação. Cada uma das fases tem suas características e objetivos próprios conforme segue: 
Esta fase objetiva envolver o aprendiz de modo a fornecer motivação para iniciar o processo de aprendizagem. Requer considerar basicamente quatro focos: I. Sondagem do conhecimento prévio; II. Identificação das concepções alternativas; III. Fornecer estímulos motivadores e indutores do interesse; IV. Desenvolver questionamentos científicos.

Para alcançar os objetivos da fase Engajar são propostas perguntas como por exemplo: (1) O que você sabe sobre...? (2) O que você viu assim? (3) Do que você ouviu falar... que você não tem certeza se é verdade? (4) 0 que você gostaria de investigar em relação a...? (Marshall et al, 2008).

Apesar dos questionamentos intencionais serem considerados importantes e necessários para o aprendizado do aluno nesta fase do processo, o autor os considera insuficientes para avaliar se os alunos estão aptos para seguir para a próxima fase, a fase Explorar. Assim mesmo que se considere a importância dos questionamentos, para o autor, eles não exigem que os alunos se envolvam na experiência de aprendizado.

Neste sentido, para conseguir o investimento dos estudantes no processo de aprendizagem são propostas a incorporação da reflexão metacognitiva com questionamentos eficazes que também podem ser apropriados à avaliação formativa.

Assim, para a fase de engajamento, o autor sugere cinco estratégias metacognitivas: I. Cadernos de ciências; II. Desenhos; III. Brainstorming (debates); IV. Exercícios de aquecimento; V. Gráficos de KWHL.

Além da incorporação da reflexão metacognitiva, será incorporado, como previsto na própria estratégia, as avaliações formativas. Estas são críticas e fornecem o feedback necessário para que o professor decida sobre seguir em frente ou permanecer na fase de engajamento. As avaliações formativas para a fase de engajamento podem incluir pré-testes, sondagens formativas e/ou gráficos KWHL (ZEE et al, 2001). Este funciona como um organizador gráfico que auxilia no aprendizado, tornando o relativamente mais fácil. As perguntas que compõem estes gráficos podem ser por exemplo. 1 . 0 que eu sei? (Know); 2. O que eu quero saber? (Want); 3. Como e descubro? (How) e 4. O que eu aprendi? (Learn) Observe o quadro 1:

Quadro 1: Esquema autoexplicativo do Gráfico KWHL

\begin{tabular}{|c|c|c|c|}
\hline K & W & H & L \\
\hline KNOW & WANT & HOW & LEARN \\
\hline $\begin{array}{c}\text { What do I know? } \\
\text { O que eu sei? }\end{array}$ & $\begin{array}{c}\text { What I want know? O } \\
\text { que eu quero saber? }\end{array}$ & $\begin{array}{c}\text { How do I find out more? } \\
\text { Como descubro mais? }\end{array}$ & $\begin{array}{c}\text { What I have learned } \\
\text { O que eu aprendi? }\end{array}$ \\
\hline & & & \\
\hline
\end{tabular}

Fonte: Arquivo dos autores

No quadro 2 apresentamos o template de aplicação da fase de Engajamento do modelo instrucional 4E X2: 
Ressaltamos que os itens da primeira linha, (Foco principal, objetivos, materiais, fontes, etc.) do quadro serão os mesmos para as quatro fases (engajamento, exploração, explicação e extensão) para um determinado assunto.

Quadro 2: Template da fase engajamento da 4E X 2

Foco principal ou questão essencial:

Objetivos:

Materiais:

Fontes / Referências / Materiais Auxiliares:

ENGAJAMENTO

Opções aplicáveis: () Conhecimento prévio; () Equívoco; () Motivação/interesse;

() desenvolvimento da questão científica

\begin{tabular}{|l|l|l|}
\hline \multicolumn{1}{|c|}{$\begin{array}{c}\text { Reflexão } \\
\text { metacognitiva }\end{array}$} & \multicolumn{1}{|c|}{ Estrutura central no Engajamento } & Contexto da avaliação \\
\hline R1: & Questões representativas: & () Centrada no \\
R2: & () O que você sabe sobre...? & conhecimento \\
Outro: & () O que você viu assim? & () Centrada no processo \\
& () O que estudamos pode se aplicar aqui? & () Centrado na habilidade \\
& () Do que você ouviu falar... que você não tem & () Individual \\
& certeza se é verdade ou não? & () Pequeno grupo \\
& () O que te intriga/interessa...? & () Desempenho da classe \\
& () O que é confuso sobre...? & A1: \\
& () Que perguntas você tem sobre...? & A2: \\
& () O que você gostaria de investigar em relação a...? & Outro: \\
\hline \multicolumn{2}{|l}{} \\
\hline \multicolumn{2}{|l}{ Tempescrição do engajamento: } \\
\hline \multicolumn{2}{|l}{ Decisão pós-sessão: () Prosseguir; () Revisão rápida; () Retificar } \\
\hline
\end{tabular}

Fonte: Adaptado de Marshall et al (2008).

\section{Explorar (Explore)}

Uma vez que os professores percebam o envolvimento (engajamento) efetivo dos alunos, estes podem ser direcionados para a fase de exploração. Os aspectos críticos da fase de exploração permitem aos alunos o desenvolvimento de previsão de situações, projeção de contextos, coleta de informações e teste de hipóteses. (Marshall et al, 2008)

Como exemplos de questionamentos eficazes capazes de orientar os alunos nestes aspectos temos: (1) E se ...? (2) Como você pode estudar melhor este problema? (3) O que acontece quando...? (4) Quais dados / informações você precisa coletar? (5) Por que você escolheu seu método para estudar o problema?

Na fase de exploração, assim como na fase de engajamento, a reflexão metacognitiva e a avaliação formativa são essenciais para manter os alunos direcionados ao longo do caminho do aprendizado.

Marshall et al (2008) sugere também nesta fase o uso do gráfico KWHL, especificamente o item " $\mathrm{H}$ ", parte em que o aluno se questiona: "Como eu estudo efetivamente esta questão ou problema?" Nesta fase os professores podem induzir que os alunos identifiquem e/ou elaborem onde estão as (c) (1) () 
fraquezas e confusões em seus planos de estudo. Os autores consideram que a unificação entre a reflexão metacognitiva, o modelo instrucional de investigação e a avaliação formativa durante esta fase, pode intencionalmente, encorajar uma compreensão mais profunda.

No quadro 3 apresentamos o template da fase de exploração do modelo instrucional 4E X 2 .

Quadro 3: Template da fase exploração da 4E X 2

\begin{tabular}{|c|c|c|}
\hline \multicolumn{3}{|c|}{$\begin{array}{l}\text { Foco principal ou questão essencial: } \\
\text { Objetivos: } \\
\text { Materiais: } \\
\text { Fontes / Referências / Materiais Auxiliares: }\end{array}$} \\
\hline \multicolumn{3}{|c|}{ EXPLORAÇÃO } \\
\hline Opções aplic & s: () Prever; ( )Projeto; () Teste; () Coleta; () Ra & \\
\hline $\begin{array}{c}\text { Reflexão } \\
\text { metacognitiva }\end{array}$ & Estrutura central na Exploração & Contexto da avaliação \\
\hline $\begin{array}{l}\text { R3: } \\
\text { R4: } \\
\text { Outro: }\end{array}$ & $\begin{array}{l}\text { Questões representativas: } \\
\text { () E se...? } \\
\text { () O que você espera que aconteça? Por quê? } \\
\text { () Como você pode estudar melhor este problema? } \\
\text { () O que você precisa coletar? } \\
\text { () Como você vai organizar suas informações? } \\
\text { () Quantos dados/informações você precisa coletar? } \\
\text { () Quais são as mudanças que você percebeu em...? } \\
\text { () Dê outros: }\end{array}$ & $\begin{array}{l}\text { () Centrada no conhecimento } \\
\text { () Centrada no processo } \\
\text { () Centrado na habilidade } \\
\text { () Individual } \\
\text { () Pequeno grupo } \\
\text { () Desempenho da classe } \\
\text { A3: } \\
\text { A4: } \\
\text { Outro: }\end{array}$ \\
\hline
\end{tabular}

Tempo necessário para completar a exploração:

Descrição da exploração:

Decisão pós-sessão: () Prosseguir; ( ) Esclarecer; () Retificar; ( ) Reengajar.

Fonte: Adaptado de Marshall et al (2008).

\section{Explicar (Explain)}

Nesta fase 0 aluno começa a perceber 0 alinhamento entre 0 conhecimento prévio e concepções alternativas da fase de Engajamento, com as descobertas da fase de Exploração. Cabe ressaltar a importância da evocação de conhecimentos prévios, uma vez que, segundo Moreira, (2012), a aprendizagem passa a ser significativa quando há interação entre conhecimentos prévios e conhecimentos novos.

Marshall et al (2008) considera que apesar do modelo de instrução de investigação ser altamente dinâmico, é fundamental que a fase de exploração preceda a fase de explicação. De modo contrario, correr-se-ia o risco de "empurrar" o estudante para situações de aprendizado passivo sem o desafio de confrontar dificuldades no conhecimento prévio ou concepções alternativas.

Nesta fase, o conteúdo torna-se central, pois as habilidades do processo são utilizadas para dar suporte a habilidades de ordem superior como interpretação justificativa e análise. 
A evolução da fase de explicação depende do desenvolvimento de alguns pontos que são considerados centrais: 1. Interpretação dos dados encontrados; 2. Busca pelo embasamento das discussões em relação à temática abordada; 3. Comunicação das conclusões (escrita, oral, ou com auxílio de tecnologias de comunicação) e 4. Busca por explicações alternativas para os achados. O quadro 4 apresenta a lista de verificação da fase de explicação.

Além das questões apresentadas no quadro 4, referentes ao modelo de instrução de investigação, Marshall propõe para esta fase, a reflexão metacognitiva através da conclusão dos gráficos de KWHL iniciados nas fases anteriores e ciclos de POE (prever, observar, explicar) de Hong et al (2014). $\mathrm{Na}$ conclusão das estratégias de reflexão metacognitiva, são sugeridas questões como, por exemplo: "O que você aprendeu? Explique seus resultados."

Quadro 4: Template: fase explicação da 4E X 2

\begin{tabular}{|c|c|c|}
\hline \multicolumn{3}{|c|}{$\begin{array}{l}\text { Foco principal ou questão essencial: } \\
\text { Objetivos: } \\
\text { Materiais: } \\
\text { Fontes / Referências / Materiais Auxiliares: }\end{array}$} \\
\hline \multicolumn{3}{|c|}{ EXPLICACÁO } \\
\hline \multicolumn{3}{|c|}{$\begin{array}{l}\text { Opções aplicáveis: () Interpretar; () Evidência; () Comunicar; () Alt. Explicações; } \\
\text { () Verificar; () Justificar; () Analisar. }\end{array}$} \\
\hline $\begin{array}{c}\text { Reflexão } \\
\text { metacognitiva }\end{array}$ & Estrutura central na explicação & $\begin{array}{l}\text { Contexto da } \\
\text { avaliação }\end{array}$ \\
\hline $\begin{array}{l}\text { R5: } \\
\text { R6: } \\
\text { Outro: }\end{array}$ & $\begin{array}{l}\text { Questões representativas: } \\
\text { () O que aconteceu? } \\
\text { ()) Quais mudanças você percebeu? } \\
\text { () Quais visuais ajudam a explicar suas descobertas? Explique-os. } \\
\text { () O que surpreendeu/intrigou você? O que ainda é confuso? } \\
\text { () Como isso é semelhante ou diferente de...? } \\
\text { () Que padrão (s) você percebeu? } \\
\text { () Como isso se aplica ao que aprendemos antes? } \\
\text { () Explique o que aconteceu. } \\
\text { () O que foi aprendido? } \\
\text { () Quais evidências você tem para sua declaração? } \\
\text { () Como você explicaria? } \\
\text { () Qual tendência os dados mostram? } \\
\text { ()) Como sua ideia é diferente de...? } \\
\text { () O que você quer dizer quando diz...? } \\
\text { () Você concorda com...Por que não? } \\
\text { () Onde você encontrou um fenômeno semelhante? } \\
\text { () O que você acha que vai acontecer se... } \\
\text { ()) De outros: }\end{array}$ & $\begin{array}{l}\text { ()) Centrada no } \\
\text { conhecimento } \\
\text { () Centrada no } \\
\text { processe } \\
\text { () Centrado na } \\
\text { habilidade } \\
\text { ()) Individual } \\
\text { () Pequeno } \\
\text { grupo } \\
\text { () Desempenho } \\
\text { da classe } \\
\text { A5: } \\
\text { A6: } \\
\text { Outro: }\end{array}$ \\
\hline \multicolumn{3}{|c|}{ Tempo necessário para completar a explicação: } \\
\hline \multicolumn{3}{|c|}{ Descrição da explicação: } \\
\hline \multicolumn{3}{|c|}{$\begin{array}{l}\text { Decisão pós-sessão: (w) Prosseguir; ( ) Reengajar; ( ) Reexplorar; ( ) Retificar; ( ) solicitar } \\
\text { esclarecimentos dos alumos }\end{array}$} \\
\hline
\end{tabular}

Fonte: Adaptado de Marshall et al (2008)

Complementando as atividades que compõem o modelo instrutivo de pesquisa e as estratégias de reflexão metacognitiva, na fase de explicação, também se propõe a avaliação formativa. Neste caso, com a inclusão de relatórios de laboratório, discussões e apresentações de resultados. 
Para que esta atinja o status de Avaliação Formativa com ênfase no processo de aprendizado sobre o produto, Marshall sugere a possibilidade de reenvio dos trabalhos pelos alunos, após a orientação para a revisão, com base na revisão por pares. Toma-se como objetivo a "compreensão conceitual e a compreensão da investigação científica - não se os alunos podem preencher uma planilha adequadamente." (MARSHALL ET AL, 2008, p. 510, tradução nossa).

\section{Estender (extend)}

A consolidação da compreensão conceitual e o desenvolvimento de uma representação mental permanente ocorrem a partir do momento em que os alunos têm maiores oportunidades para aplicarem seus conhecimentos em contextos autênticos e significativos (MARSHALL ET AL, 2008). Desta forma, a experiência de desequilíbrio provocada nas fases de engajamento e exploração começa a tomar forma. Neste momento, a compreensão e o conhecimento adquiridos e articulados durante a fase de explicação serão aplicados a novas situações.

Durante a fase de extensão os alunos são solicitados a elaborar, transferir, generalizar o conhecimento para novas situações. O quadro 5 apresenta as questões propostas para resta fase.

Quadro 5: Template: fase extensão da 4E X 2

\begin{tabular}{|c|c|c|}
\hline \multicolumn{3}{|c|}{$\begin{array}{l}\text { Foco principal ou questão essencial: } \\
\text { Objetivos: } \\
\text { Materiais: } \\
\text { Fontes / Referências / Materiais Auxiliares: }\end{array}$} \\
\hline \multicolumn{3}{|c|}{ EXTENSÄO } \\
\hline \multicolumn{3}{|c|}{ Opções aplicáveis: () Aplicar; () Elaborar; () Transferir; () Generalizar; } \\
\hline $\begin{array}{c}\text { Reflexão } \\
\text { metacognitiva }\end{array}$ & Estrutura central na extensão & Contexto da avaliação \\
\hline $\begin{array}{l}\text { R7: } \\
\text { R8: } \\
\text { Outro: }\end{array}$ & $\begin{array}{l}\text { Questões representativas: } \\
\text { () O que aconteceria se ...? } \\
\text { () Como você acha que ... se aplica a ...? } \\
\text { () Explique de outro ponto de vista. } \\
\text { () Como isso pode ser usa do no mundo real? } \\
\text { () Que questões / problemas ainda não foram } \\
\text { resolvidos? } \\
\text { () Que decisões precisam ser feitas? } \\
\text { () Quais consequências/beneficios/ riscos } \\
\text { acompanhamcertas decisões? }\end{array}$ & $\begin{array}{l}\text { () Centrada no conhecimento } \\
\text { () Centrada no processo } \\
\text { () Centrado na habilidade } \\
\text { () Individual } \\
\text { () Pequeno grupo } \\
\text { () Desempenho da classe } \\
\text { A7: } \\
\text { A8: } \\
\text { Outro: }\end{array}$ \\
\hline \multicolumn{3}{|c|}{ Tempo necessário para completar a extensão: } \\
\hline \multicolumn{3}{|c|}{ Descrição da extensão: } \\
\hline $\begin{array}{l}\text { Decisão pós-ses } \\
\text { () Outra extens }\end{array}$ & () Debrief; () Reengajar; () Reexplorar; & citar esclarecimentos dos alun \\
\hline
\end{tabular}

Fonte: Adaptado de Marshall et al (2008). 


\section{A metacognição na 4E X 2}

$\mathrm{Na} 4 \mathrm{E} \times 2$ a interação entre os elementos que tratam da reflexão e os elementos que tratam da metacognição foi chamada por Marshall et al (2008) de reflexão metacognitiva. Os autores propõem que os aprendizes em determinado momento da execução da tarefa deverão explicar as estratégias usadas para a resolução de um problema científico (reflexão), e na sequência fornecer detalhes sobre os próprios processos de pensamento durante a seleção e implementação dessas estratégias (metacognição).

Neste contexto é possível observar a interface entre as atividades desenvolvidas e conceitos da teoria metacognitiva. Ao retomar as estratégias usadas para a solução do problema, o aprendiz deverá tomar consciência sobre os seus próprios pensamentos, ou seja, deverá exercitar o conhecimento daquilo que ele conhece (FLAVELL, 1976; 1979). E o fornecimento de detalhes sobre os próprios processos de pensamento durante a seleção das estratégias, exige exercício do controle executivo regulador que inclui planificação, monitoramento e avaliação. (BROWN, 1978; 1987; FLAVELL, 1976)

O constructo da avaliação formativa apresenta vantagem sobre outros tipos de avaliação. Isto ocorre justamente devido a sua função de formação sobre indivíduo. Através dele o aprendiz torna-se capaz de reconhecer sucessos, inconsistências e equívocos de modo que possam se orientar sobre como proceder e corrigir suas atitudes em um empreendimento de aprendizagem ou solução de um problema durante o processo (ANDRIOLA; ARAÚJO 2018; BLACK; WILIAM 1998; HOFFMAN, 1994; VIEIRA; LIMA, 2019).

Observa-se que avaliação formativa também apresenta elementos da metacognição uma vez que reconhecer sucessos, inconsistências e equívocos também envolve conhecimento sobre o próprio conhecimento, tanto na variável pessoa (conhecimento capacidades e limitações próprias e dos outros), quanto na variável tarefa, relacionada ao conhecimento sobre a natureza da informação, por exemplo, em relação às suas demandas e exigências e sobre como ela é e o que envolve seu entendimento e realização. Além do mais, relaciona-se com a variável estratégia que indica quais são os processos, procedimentos e ações que permitem que o sujeito atinja seus objetivos com maior eficácia. (FLAVELL; WELLMAN, 1977; FLAVELL, 1979; ROSA; VILAGRÁ, 2020).

Outro constructo da 4E X 2, a Instrução por investigação, apresenta quatro fases, respectivamente Engajar, Explorar, Explicar e Estender. Cada uma das fases apresenta questionamentos específicos que orientam o aprendiz durante o processo investigativo. Alguns destes questionamentos podem estimular o desenvolvimento de elementos do pensamento metacognitivo conforme descritos a seguir. Cabe ressaltar que a reflexão metacognitiva e a avaliação formativa podem ser aplicadas em todas as fases da instrução por investigação.

A fase de engajamento propõe provocar perturbação e atrair a atenção do aprendiz. Tem o objetivo de fornecer motivação e dar início ao processo de 
aprendizagem. Analisando algumas questões representativas desta fase: $O$ que você sabe sobre...? O que você viu assim? O que te intriga/interessa...? Que perguntas você tem sobre...? - É possível observar o elemento metacognitivo ou variável pessoa do componente metacognitivo conhecimento do conhecimento (FLAVELL; WELLMAN, 1977) uma vez que estimula o aluno a pensar sobre o que ele sabe e pensar sobre o que ele pensa. $E$ as questões representativas da fase de engajamento podem ser comparadas com as perguntas metacognitivas: Identifica este assunto com outro já estudado? Apresenta conhecimento sobre o assunto? Qual o sentimento em relação a este conhecimento? - propostas por Rosa (2011) e Rosa e Villagrá (2020).

Cabe ressaltar que as questões representativas desta fase também estão relacionadas com a aprendizagem significativa de Ausubel (2003) quando busca atribuir significados a novos conhecimentos considerando a existência de conhecimentos prévios.

A próxima fase, Exploração, caracteriza-se pela coleta de informações com o objetivo de buscar a compreensão daquilo que está sendo investigado. Assim o aprendiz pode mergulhar em aspectos críticos como previsão de situações, projeção de contextos, testes de hipóteses entre outros.

Nesta fase, além da componente conhecimento do conhecimento na variável tarefa, o aprendiz exercita o elemento da planificação do controle executivo autorregulador de Flavell e Wellman (1977). Isso pode ser observado nas questões representativas propostas: Como você pode estudar melhor este problema? Como você vai organizar suas informações? Quantos dados/informações você precisa coletar? Estas questões fazem interface com as perguntas metacognitivas propostas por Rosa (2011) e Rosa e Villagrá (2020): Identifica o que é preciso para resolvê-la? Como organizar as informações apresentadas na atividade? - Organizadas e classificadas na tabela 1.

$\mathrm{Na}$ fase de explicação destaca-se a interpretação dos dados, embasamento nas discussões, comunicação das conclusões e finalmente a busca por explicações alternativas para as descobertas. As questões representativas: O que aconteceu? Quais mudanças você percebeu? Quais visuais ajudam a explicar suas descobertas? O que surpreendeu/intrigou você? O que ainda é confuso? Podem estimular no aprendiz o controle executivo autorregulador (BROWN, 1978; 1987; FLAVELL; WELLMAN, 1977;) e fazem interface com as perguntas metacognitivas de Rosa (2011) e Rosa e Villagrá (2020), Qual o resultado da atividade? Tem consciência do conhecimento adquirido com a realização da atividade? Classificadas como elemento metacognitivo de avaliação, conforme apresentado na tabela 1 .

Por fim a fase da extensão, quando as descobertas adquiridas até agora ficam passíveis de aplicação em novas situações. Nesta fase o aprendiz é incentivado a aplicar, elaborar, transferir e generalizar os conhecimentos adquiridos, em novas situações de modo a extrapolar o que aprenderam. (Maraglia, 2018). 
Observe que tanto na reflexão metacognitiva quanto na avaliação formativa e nos questionamentos da instrução por investigação, encontramos elementos da metacognição uma vez que o aluno é levado a exercitar o conhecimento sobre 0 próprio conhecimento e 0 controle executivo autorregulador (BROWN, 1978; 1987; FLAVELL, 1976; FLAVELL; WELLMAN 1977;). Além do mais, as questões representativas fazem interface com as perguntas metacognitivas de Rosa (2011) e Rosa e Villagrá (2020) podendo funcionar como comandos metacognitivos (PETERS; KITSANTAS, 2010) que se concentram nas dúvidas, equívocos, anseios, estratégias para a solução de problemas e para a comunicação das conclusões dos alunos em relação ao tema.

\section{Considerações finais}

A apresentação dos constructos instrução por investigação, reflexão metacognitiva e avaliação formativa e a forma como foi descrita, permite orientar o professor em uma instrução envolvente e interativa de modo que o aluno possa parar, interagir, repensar e refletir sobre seus processos de aprendizagem, além de estimular o registro de seus padrões de solução e a busca por outras opções de solução.

Encontramos potencialidades para a execução, especialmente pela forma pragmática e sistemática oferecida pelos templates aos quais se organizam as fases da instrução com suas respectivas questões representativas e pelas sugestões de estratégias para reflexão metacognitiva e avaliação formativa. Desta forma, consideramos os templates como importantes modelos para o planejamento e execução aos leitores interessados em produzir ou reproduzir a 4E X 2. Também observamos que a EEM 4E X 2 mostra-se flexível o suficiente para que professores, de forma criativa e dinâmica, possam adaptar experimentos atividades aos seus objetivos e de acordo com suas realidades.

Compreendemos a 4E X 2 como uma importante estratégia de ensino metacognitivo uma vez que pode ser planejada e implementada de modo em que sejam trabalhados conteúdos ao mesmo tempo que a metacognição e a aprendizagem autorregulada é estimulada.

Encontramos tanto no constructo da reflexão metacognitiva quanto no constructo da avaliação formativa, propostos na 4E X 2, relações diretas com o exercício da metacognição. Assim, tanto de forma independente quanto pela fusão dos constructos, observamos a relação e o exercício da metacognição sob as perspectivas da componente conhecimento do conhecimento e da componente controle executivo autorregulador.

Neste contexto, o aprendiz ao executar a tarefa pode ser estimulado através das estratégias de reflexão metacognitiva e da avaliação formativa a refletir sobre o próprio conhecimento além de planejar, monitorar e avaliar o processo de aprendizagem para a solução do problema.

No constructo da instrução por investigação, observamos potencial de desenvolvimento metacognitivo nas questões representativas. Estas questões 
foram associadas às perguntas metacognitivas propostas por e organizadas por Cleci Rosa e Villagrá como elementos metacognitivos de pessoa, tarefa e estratégia da componente conhecimento do conhecimento e como elementos de planejamento, monitoração e avaliação da componente do controle executivo e autorregulador, propostos e detalhados anteriormente por Flavell, Wellman e Brown.

Assim, as questões representativas podem funcionar como orientações metacognitivas que estimulam os processos de pensamento sobre o que 0 aprendiz sabe, sobre a natureza das informações que possui e sobre as estratégias necessárias para a solução do problema. Além do mais, as questões representativas podem estimular no aprendiz a capacidade de planejar e monitorar ações e avaliar seu processo de aprendizagem.

Este estudo também apontou possibilidades e valiosas informações para a utilização da estratégia de ensino metacognitiva - 4E X 2 em sala de aula. Assim sugerimos para os próximos passos a formulação, aplicação e avaliação empírica de atividades baseadas nesta estratégia de acordo com a realidade das escolas brasileiras.

Em um sentido mais amplo, reconhecemos a necessidade de desenvolvimento de novas pesquisas de avaliação não só das relações com a teoria metacognitiva, mas sobre a exequibilidade desta e de outras Estratégias de Ensino Metacognitivas disponíveis na literatura científica. E assim buscar a aproximação entre que tem sido produzido no contexto acadêmico e o que é possível reproduzir no ambiente escolar, especialmente brasileiro.

\section{Agradecimentos}

O presente trabalho foi realizado com apoio da Coordenação de Aperfeiçoamento de Pessoal de Nível Superior - Brasil (CAPES) - Código de Financiamento 001, do Instituto Federal de Educação Ciência e Tecnologia do Espírito Santo e da Universidade Federal do Rio de Janeiro.

\section{REFERÊNCIAS}

ADLER, Mortimer Jerome; DOREN, Charles Van. Como ler livros: O Guia Clássico para a Leitura Inteligente. São Paulo: É Realizações, 2010.

AUSUBEL, David P. Aquisição e retenção de conhecimentos: uma perspectiva cognitiva. Lisboa: Plátano, v. 1, 2000.

ANDRIOLA, Wagner Bandeira; ARAÚJO, Adriana Castro. Potencialidades da avaliação Formativa e Somativa. Revista Eletrônica Acta Sapientia, v. 5, n. 1, p. 15-15, 2018. 
ASCHBACHER, Pamela.; ALONZO, Alicia. Using science notebooks to assess students' conceptual understanding. American Educational Research Association, San Diego, 2004.

BROWN, Ann Lesley. Knowing when, where, and how to remember: A problem of metacognition. Advances in instructional psychology, v. 1, n. 1, p. 77$165,1978$.

BROWN, Ann Lesley. Metacognition, executive control, self-regulation, and other more mysterious mechanisms. Em F. E. Weinert \& R. Kluwe (Orgs.), Metacognition, motivation, and understanding (pp. 1-16). Hillsdale, N. J.: Erlbaum, 1987.

BLACK, Paul; WILIAM, Dylan. Assessment and classroom learning. Assessment in Education: principles, policy \& practice, v. 5, n. 1, p. 7$74,1998$.

CHI, Michelene; BASSOK, Mirian; LEWIS, Matthew; REIMAN, Peter; GLASER, Robert. Self-Explanations: How Students Study and Use Examples in Learning to Solve Problems. Cognitive Science, [s.I.], v. 13, no 2, p. 145-182, 1989.

CLEARY, Timothy J.; PLATTEN, Peter. Examining the correspondence between self-regulated learning and academic achievement: A case study analysis. Education Research International, v. 2013, 2013.

CLEOPHAS, Maria das Graças.; FRANCISCO, Welington. Metacognição e o ensino e aprendizagem das ciências: uma revisão sistemática da literatura (RSL). Amazônia: Revista de Educação em Ciências e Matemáticas, v. 14, n. 29, p. 10-26, 2018.

DUNLOSKY, John., \& HERTZOG, Christopher. Aging and deficits in associative memory: What is the role of strategy use? Psychology and Aging, 13, 597$607,1998 a$.

ESTEBAN, Maria. Pesquisa qualitativa em educação: Fundamentos e tradições. [S. I.]: Artmed, 2010.

FLAVELL, John Hurley. Metacognitive aspects of problem solving. In: L. B. Resnik (Ed.). The Nature of Intelligence. Hillsdale: Lawrence Erlbaum, 1976.

FLAVELL, John Hurley; WELLMAN, Henry. Metamemory. In Robert V. Kail, \& John W. Hagen (Eds), Perspectives on the development of memory and cognition (pp.3-33). New Jersey: Lawrence Erlbaum Associates, 1977. 
FLAVELL, John Hurley. Metacognition and cognitive monitoring: A new area of cognitive-developmental inquiry. American Psychologist, [s.I.], v. 34, no 10, p. 906-911, 1979.

FLAVELL, John Hurley; MILLER, Patricia H.; MILLER, Scott. A.

Desenvolvimento cognitivo. Porto Alegre: Artmed, 1999.

GASQUE, Kelley Cristine Gonçalves Dias. Indicador de atividade reflexiva e teoria fundamentada: o pensamento reflexivo na busca e no uso da informação. Transinformação, v. 23, n. 1, p. 39-49, 2011.

HOFFMANN, Jussara Maria Lerch. Avaliação mediadora: uma relação dialógica na construção do conhecimento. Avaliação do rendimento escolar. São Paulo: FDE, p. 51-9, 1994.

HONG, Jon-Chao; HWANG, Ming Yueh; LIU, Ming Chou; HO, Huei Yin; CHEN, Yi Ling et al. Using a "prediction-observation-explanation" inquiry model to enhance student interest and intention to continue science learning predicted by their Internet cognitive failure. Computers \& Education, v. 72, p. 110120, 2014.

LEITE, Eliana Alves Pereira; DARSIE, Marta Maria Pontin. Implicações da metacognição no processo de aprendizagem da Matemática. Revista Eletrônica de Educação, v. 5, n. 2, p. 179-191, 2011.

LOCATELI, Solange Wagner Tópicos de Metacognição: Para aprender e ensinar melhor. 1. ed. Curitiba: Appris, 2014. 75 p. v. 1.

MARAGLIA, Pedro Henrique Estratégias de ensino metacognitivas: uma revisão sistemática de literatura. Dissertação - Núcleo de Tecnologia Educacional para a Saúde, Universidade Federal do Rio de Janeiro. Rio de Janeiro, 2018.

MARSHALL, Jeff; HORTON, Bob; SMART, Julie. 4E $\times 2$ instructional model: Uniting three learning constructs to improve praxis in science and mathematics classrooms. Journal of Science Teacher Education, v. 20, n. 6, p. 501-516, 2008.

MOREIRA, Marco Antônio. Aprendizagem significativa: a teoria e textos complementares. São Paulo: Editora Livraria da Física, 2012.

OSES, Sonia ; CARRASCO, Laura. Módulos alternativos en la enseñanza de las ciencias: estrategia didáctica orientada al logro de aprendizajes

significativos. Formación universitaria, v. 6, n. 3, p. 39-52, 2013.

PEIXOTO, Maurício de Abreu Pinto; BRANDÃO, Marcos Antônio Gomes; SANTOS, Gladis dos. Metacognição e tecnologia educacional 
simbólica. Revista Brasileira de Educação Médica, v. 31, n. 1, p. 67-80, 2007.

PETERS, Erin.; KITSANTAS, Anastasia. The Effect of Nature of Science Metacognitive Prompts on Science Students' Content and Nature of Science Knowledge, Metacognition, and SelfRegulatory Efficacy. School Science and Mathematics, v. 110, n. 8, p. 382-396, 2010.

PRATES, Admilson Eustáquio; BETARELLO, Jeferson; FINELLI, Leonardo Augusto Couto. REFLEXÕES SOBRE O ATO DE LER. Humanidades, v. 5, n. 1, 2016.

ROSA, Cleci Werner. A metacognição e as atividades experimentais no ensino de Física. Tese de Doutorado. Universidade Federal de Santa Catarina, Florianópolis, SC, 2011.

ROSA, Cleci Werner da; ALVES FILHO, José de Pinho. Estudo da viabilidade de uma proposta didática metacognitiva para as atividades experimentais em física. Ciência \& Educação (Bauru), [s.l.], v. 20, no 1, p. 61-81, 2014. ISSN: 1516-7313, DOI: https://doi.org/10.1590/1516-731320140010005

ROSA, Cleci Werner da; VILLAGRÁ, Jesús Ángel Meneses. Metacognição e Ensino de Física: Revisão de Pesquisas Associadas a Intervenções Didáticas. Revista Brasileira de Pesquisa em Educação em ciências, $p$. 581-608, 2018. DOI: https://doi.org/10.28976/1984-2686rbpec2018182581

ROSA, Cleci Werner da; ROSA, Álvaro Becker. Ensino de física por estratégias metacognitivas: análise da prática docente. Revista electrónica de investigación en educación en ciencias, v. 11, n. 1, p. 1-8, 2016.

ROSA, Cleci Werner da; VILLAGRÁ, Jesús Ángel Meneses. Questionamento metacognitivo associado à abordagem didática por indagação: análise de uma atividade de ciências no ensino fundamental. Investigações em Ensino de Ciências, v. 25, n. 1, p. 60-76, 2020.

DOI: http://dx.doi.org/10.22600/1518-8795.ienci2020v25n1p60

SHEPARDSON, Daniel; BRITSCH, Susan. The role of children's journals in elementary school science activities. Journal of Research in Science Teaching, v. 38, n. 1, p. 43-69, 2001.

STERNBERG, Robert J. Metacognition, abilities, and developing expertise: What makes an expert student?. Instructional science, v. 26, n. 1, p. 127-140, 1998.

TARRICONE, Pina. The taxonomy of metacognition. Hove; New York: Psychology Press, 2011. 
VANDEVOORT, Leslie G.; AMREIN-BEARDSLEY, Audrey; BERLINER, David C. National Board Certified Teachers andTheir Students' Achievement. education policy analysis archives, v. 12, p. 46, 2004.

VIEIRA, Luzineide de Sousa dos Santos; LIMA, Maria Loureto. Avaliação no Ensino Superior: um olhar à prática avaliativa do curso de Pedagogia da Universidade Regional do Cariri. Id on Line Rev. Mult. Psic, v. 13, n. 47, p. 1097-1122, 2019.

XAVIER, César Silva.; PEIXOTO, Mauricio Abreu Pinto Estratégias de ensino metacognitivas e o aprendizado de biologia no ensino médio: o que há na literatura? IX Encontro regional de ensino de biologia - EREBIO RJ/ES, 2019, Rio de Janeiro.

XAVIER, César Silva; PEIXOTO, Mauricio Abreu Pinto; VEIGA, Luciana Lima de Albuquerque da. Metacognição e suas ferramentas para o aprendizado.

Revista Eletrônica Debates em Educação Científica e Tecnológica, v. 10, n. 2, 2020. DOI: https://doi.org/10.36524/dect.v10i2.1337

ZEE, Emily H. Van; IWASYK, Marletta; KUROSE, Akiko; SIMPSON, Doroty; WILD, Judy. Student and teacher questioning during conversations about science. Journal of Research in Science Teaching, 38(2), 159-190, 2001.

WHITE, Barbara; FREDERIKSEN, John. A theoretical framework and approach for fostering metacognitive development. Educational Psychologist, v. 40, n. 4, p. 211-223, 2005.

WILSON, Jeni; CLARKE, David. Towards the modelling of mathematical metacognition. Mathematics Education Research Journal, v. 16, n. 2, p. 25-48, 2004. 